\title{
Identification of differentially expressed IncRNAs and mRNAs in luminal-B breast cancer by RNA-sequencing
}

Cheng-Liang Yuan ${ }^{1}$, Xiang-Mei Jiang ${ }^{1}$, Ying Yi ${ }^{2}$, Jian-Fei E1, Nai-Dan Zhang ${ }^{1}$, Xue Luo ${ }^{2}$, Ning Zou ${ }^{1 *}$, Wei Wei ${ }^{1}$ and Ying-Ying Liu ${ }^{3}$

\begin{abstract}
Background: Luminal B cancers show much worse outcomes compared to luminal A. This present study aims to screen key IncRNAs and mRNAs correlated with luminal-B breast cancer.

Methods: Luminal-B breast cancer tissue samples and adjacent tissue samples were obtained from 4 patients with luminal-B breast cancer. To obtain differentially expressed mRNAs (DEmRNAs) and IncRNAs (DEIncRNAs) between luminal-B breast cancer tumor tissues and adjacent tissues, RNA-sequencing and bioinformatics analysis were performed. Functional annotation of DEmRNAs and protein-protein interaction networks (PPI) construction were performed. DEmRNAs transcribed within a $100 \mathrm{~kb}$ window up- or down-stream of DElncRNAs were searched, which were defined as cis nearby-targeted DEmRNAs of DElncRNAs. DElncRNA-DEmRNA co-expression networks were performed. The mRNA and IncRNA expression profiles were downloaded from The Cancer Genome Atlas (TCGA) database to validate the expression patterns of selected DEmRNAs and DElncRNAs.
\end{abstract}

Results: A total of 1178 DEmRNAs and 273 DEIncRNAs between luminal-B breast cancer tumor tissues and adjacent tissues were obtained. Hematopoietic cell lineage, Cytokine-cytokine receptor interaction, Cell adhesion molecules (CAMs) and Primary immunodeficiency were significantly enriched KEGG pathways in luminal-B breast cancer. FN1, EGFR, JAK3, TUBB3 and PTPRC were five hub proteins of the PPI networks. A total of 99 DElncRNAs-nearby-targeted DEmRNA pairs and 1878 DEIncRNA-DEmRNA co-expression pairs were obtained. Gene expression results validated in TCGA database were consistent with our RNA-sequencing results, generally.

Conclusion: This study determined key genes and IncRNAs involved in luminal-B breast cancer, which expected to present a new avenue for the diagnosis and treatment of luminal-B breast cancer.

Keywords: Luminal-B breast cancer, mRNA, Long non-coding RNA (IncRNA), RNA-sequencing

\section{Background}

Breast cancer is the leading cause of cancer-related death in women, both overall and in less developed countries (1). It is a heterogeneous disease with regard to molecular alterations, cellular composition, and clinical outcome, both between tumor subtypes and within a single tumor, which were commonly defined by gene expression profiling as four main subtypes including luminal A, luminal B, HER-2 enriched and basal-like, (2-

\footnotetext{
* Correspondence: deylab@163.com

'Department of Clinical Laboratory, People's Hospital of Deyang City, No.

173, Taishan North Road, Jingyang District, Deyang 618000, Sichuan, China

Full list of author information is available at the end of the article
}

4). Luminal B breast cancer is unique with regard to somatic point mutations, the profile of gene copy number alterations (CNAs), and DNA methylation (5). Expression profiles and gene sets, with prognostic, predictive functions, or both for patients with breast cancer, have been identified in multiple studies (6). Although both luminal-A and luminal-B breast cancers are ER-positive, luminal-B cancers showed worse outcomes as compared to luminal-A cancers $(7,8)$. Therefore, it is urgent to discover novel biomarkers with prognostic and predictive functions for luminal B breast cancer that can be therapeutically targeted.

(c) The Author(s). 2019 Open Access This article is distributed under the terms of the Creative Commons Attribution 4.0 International License (http://creativecommons.org/licenses/by/4.0/), which permits unrestricted use, distribution, and reproduction in any medium, provided you give appropriate credit to the original author(s) and the source, provide a link to the Creative Commons license, and indicate if changes were made. The Creative Commons Public Domain Dedication waiver (http://creativecommons.org/publicdomain/zero/1.0/) applies to the data made available in this article, unless otherwise stated. 
Table 1 Patient characteristics

\begin{tabular}{|c|c|c|c|c|c|c|c|}
\hline \multirow[t]{2}{*}{ Index } & \multirow{2}{*}{$\begin{array}{l}\text { Age } \\
\text { Range }\end{array}$} & \multicolumn{3}{|c|}{ TNM stage } & \multirow[t]{2}{*}{ ER } & \multirow[t]{2}{*}{ PR } & \multirow{2}{*}{$\begin{array}{l}\text { HER- } \\
2\end{array}$} \\
\hline & & $\bar{T}$ & $\mathrm{~N}$ & M & & & \\
\hline Case 1 & $46-56$ & 3 & 0 & 0 & $30 \%$ & $10 \%$ & - \\
\hline Case 2 & & 2 & 1 & 0 & $80 \%$ & $60 \%$ & + \\
\hline Case 3 & & 2 & 1 & 0 & $40 \%$ & $30 \%$ & - \\
\hline Case 4 & & 2 & 1 & 0 & $40 \%$ & $20 \%$ & - \\
\hline
\end{tabular}

$T N M$ stage Tumor-node-metastasis stage, $E R$ ertrogen receptor, $P R$ progestrone receptor, HER-2 human epidermal growth factor receptor-2

With advances in high-throughput technology, it is discovered that human transcriptome mainly consists of non-coding RNAs (ncRNAs) with limited or no protein-coding capacity $(9,10)$. Long non-coding RNAs (lncRNAs), with over 200 nucleotides base long, attracts more attention and has been widely linked with various diseases, including cancers (1114). The lncRNAs exert momentous roles in multiple cellular processes at transcriptional and posttranscriptional regulation level through transcriptional interference and histone modifications $(15,16)$. The higher expression of SPRY4-IT1 was reported to modulate apoptosis and invasion in melanoma (17). LncRNA UCA1a (CUDR) may promote proliferation and tumorigenesis in human bladder cancer (18).

In this study, differentially expressed lncRNAs (DElncRNA) and mRNAs (DEmRNAs) in tumor tissues of patients with luminal-B breast cancer were identified by RNA-sequencing. Subsequently, protein-protein interaction (PPI) networks of DEmRNAs were conducted. Identification of cis nearby-targeted DEmRNAs of DElncRNAs and construction of DElncRNADEmRNA co-expression networks were performed. In this light, we expect this study could represent a new avenue to improve the understanding of the pathogenesis and be helpful for treatment of luminal B breast cancer.

\section{Methods}

\section{Patients and samples}

Luminal-B breast cancer tissue samples and adjacent tissue samples were obtained from 4 patients with luminalB breast cancer in People's Hospital of Deyang City, which were free of treatment. The detailed characteristics of patients are displayed in Table 1. Written informed consent about the use of these samples was obtained from each patient. All procedures performed in this study was in accordance with the ethical standards of the ethics committee of People's Hospital of Deyang City (2017-045) and with the 1964 Helsinki declaration and its later amendments or comparable ethical standards.

\section{RNA isolation and sequencing}

According to the manufacturer's protocol, RNA was extracted with PAXgene blood RNA kit (PreAnalytiX

Table 2 Top 10 up- and down-regulated DEmRNAs between luminal-B breast cancer tumor tissues compared with adjacent tissues

\begin{tabular}{lllll}
\hline ID & Symbol & $\log _{2}$ FC & p-value & Regulation \\
\hline ENSG00000143556 & S100A7 & 8.127250867 & 7.08 E-10 \\
ENSG00000171951 & SCG2 & 4.46199863 & $7.47 E-09$ & up \\
ENSG00000163993 & S100P & 5.865266947 & $2.43 E-08$ & up \\
ENSG00000169245 & CXCL10 & 5.755282864 & 2.57 E-08 & up \\
ENSG00000188404 & SELL & 4.205586942 & $2.63 E-08$ & up \\
ENSG00000180549 & FUT7 & 3.310463733 & $3.42 E-08$ & up \\
ENSG00000138755 & CXCL9 & 4.319459635 & $4.19 E-08$ & up \\
ENSG00000099953 & MMP11 & 4.120231544 & $1.44 E-07$ & up \\
ENSG00000184937 & WT1 & 2.831855381 & $2.43 E-07$ & up \\
ENSG00000143546 & S100A8 & 5.625786123 & $3.05 E-07$ & up \\
ENSG00000269711 & AC008763.3 & -8.573808999 & $3.62 E-11$ & down \\
ENSG00000272414 & FAM47E-STBD1 & -4.255105776 & $3.94 E-07$ & down \\
ENSG00000109846 & CRYAB & -2.409617655 & $1.98 E-06$ & down \\
ENSG00000109107 & ALDOC & -2.539792463 & $3.92 E-06$ & down \\
ENSG00000134548 & SPX & -7.064583075 & $5.21 E-06$ & down \\
ENSG00000135447 & PPP1R1A & -4.272501282 & $8.38 E-06$ & down \\
ENSG00000120049 & KCNIP2 & -3.142698521 & $1.76 E-05$ & down \\
ENSG00000270181 & BIVM-ERCC5 & -5.716264875 & $1.93 E-05$ & down \\
ENSG00000162433 & AK4 & -1.720478242 & $3.97 E-05$ & down \\
ENSG00000159387 & IRX6 & -4.343462068 & &
\end{tabular}

DEmRNAs, differentially expressed mRNAs. FC, fold change 
Table 3 Top 10 up- and down-regulated DEIncRNAs between luminal-B breast cancer tumor tissues compared with adjacent tissues

\begin{tabular}{lllll}
\hline ID & Symbol & $\log _{2}$ FC & $p$-value & Regulation \\
\hline ENSG00000235123 & DSCAM-AS1 & 6.99539501 & $2.71 E-06$ & up \\
ENSG00000273445 & AC133644.2 & 7.081588171 & $3.65 E-06$ & up \\
ENSG00000261039 & LINC02544 & 7.125899953 & $1.29 E-05$ & up \\
ENSG00000225783 & MIAT & 2.369853869 & $3.75 E-05$ & up \\
ENSG00000224950 & AL390066.1 & 2.333212245 & $7.65 E-05$ & up \\
ENSG00000270120 & AC007728.3 & 5.314205083 & $8.62 E-05$ & up \\
ENSG00000279930 & AL032819.2 & 5.526214467 & $1.62 E-04$ & up \\
ENSG00000247774 & PCED1B-AS1 & 1.82134551 & $1.78 E-04$ & up \\
ENSG00000261218 & AC099524.1 & 4.377268771 & $2.60 E-04$ & up \\
ENSG00000234261 & AL138720.1 & 4.488627977 & $2.87 E-04$ & up \\
ENSG00000253434 & LINC02237 & -6.553462635 & $1.55 E-04$ & down \\
ENSG00000250961 & AC025470.2 & -5.149166872 & $1.76 E-04$ & down \\
ENSG00000236333 & TRHDE-AS1 & -4.862423419 & $4.38 E-04$ & down \\
ENSG00000261441 & AC124068.2 & -4.394333958 & $7.43 E-04$ & down \\
ENSG00000261888 & AC144831.1 & -3.00198307 & $1.98 E-03$ & down \\
ENSG00000251660 & AC007036.3 & -2.997221226 & $2.05 E-03$ & down \\
ENSG00000260947 & AL356489.2 & -3.611436753 & $2.33 E-03$ & down \\
ENSG00000235033 & AL590999.1 & -2.980398474 & $2.69 E-03$ & down \\
ENSG00000230333 & AC004160.1 & -3.679435378 & $2.71 E-03$ & down \\
ENSG00000272701 & MESTIT1 & -3.528818654 & $2.85 E-03$ & down \\
\hline DENANAs & Affentaly exprsed & &
\end{tabular}

DElncRNAs, differentially expressed IncRNAs. FC, fold change

GmbH, Hombrechtikon, CH, Switzerland). With Agilent 2100 Bioanalyzer (Agilent RNA 6000 Nano Kit), the concentration, integrity and RNA integrity number (RIN) values of RNA were assessed. Sequencing was performed based on the Illumina Hiseq X-ten platform (Illumina, Inc., San Diego, CA, USA) with PE150 bp sequencing mode. The sequencing was done with paired-ends and $10 \mathrm{G}$ depth. With Base Calling version 0.11.4 (http://www. bioinformatics.babraham.ac.uk/projects/fastqc/), the FASTQ sequence data were acquired from the RNAsequencing data. Read QC tool in FastQC version 0.11.4 (http://www.bioinformatics.babraham.ac.uk/projects/ fastqc/) was used for the quality control of FASTQ data with $Q>30$. Trimming of raw data was performed with cutadapt version 1.16 (http://cutadapt.readthedocs.io). Reads with low quality (adaptor sequences, sequences with a quality score $<20$, and sequences with an $\mathrm{N}$ base rate of raw reads $>10 \%$ ) were removed to obtain the clean reads.

\section{Identification of DEmRNAs and DEIncRNAs}

In order to align the clean reads with the human reference genome, Ensemble GRCh38.p7 (ftp://ftp.ncbi.nlm.nih.gov/ genomes/Homo_sapiens), HISAT2 version 2.1.0 (https:// ccb.jhu.edu/software/hisat2/index.shtml) was applied. Expression of mRNAs and lncRNAs was normalized and outputted with StringTie version 1.3.3b (http://ccb.jhu. edu/software/stringtie/). Fragments per Kilobase of exon per million fragments mapped (FPKM) of lncRNAs and mRNAs were calculated with StringTie. With edgeR version 3.24 (http://www.bioconductor.org/packages/release/ bioc/html/edgeR.html), both DEmRNAs and DElncRNAs were obtained with $|\log 2 \mathrm{FC}|>1$ and $p$-value $<0.05$. By using R package "pheatmap", hierarchical clustering analysis of DElncRNAs and DEmRNAs were conducted.

\section{Functional annotation of DEmRNAs}

Functional annotation, including Gene Ontology (GO) function and Kyoto Encyclopedia of Genes and Genomes (KEGG) pathway enrichment analyses was performed with Metascape (http://metascape.org/gp/index. html). A value of $p<0.05$ was set as the cut-off for significance.

\section{Protein-protein interaction (PPI) networks construction}

Top 100 up- and down-regulated DEmRNAs were scanned with the BioGrid (http://www.uniprot.org/database/DB-0184). Then, PPI networks were visualized with Cytoscape software (version 3.5.0, http://www.cytoscape. org).

\section{Cis nearby-targeted DEmRNAs of the DElncRNAs}

DEmRNAs transcribed within a 100-kb window upstream or downstream of DElncRNAs were searched, which were defined as cis nearby-targeted DEmRNAs of DElncRNAs, to obtain the targeted DEmRNAs of DElncRNAs with cis-regulatory effects. The networks were visualized by Cytoscape software. Functional annotation of the cis nearby-targeted DEmRNAs of the DElncRNAs was performed with Metascape. A value of $p<0.05$ was set as the cut-off for significance.

\section{DElncRNA-DEmRNA co-expression networks}

To further examine the potential roles of DElncRNAs and DEmRNAs in luminal-B breast cancer, the DElncRNADEmRNA co-expression networks were constructed. DElncRNA-DEmRNA pairs with an absolute value of PCC $>0.95$ and $p<0.01$ were defined as co-expressed DElncRNA-DEmRNA pairs. By using Cytoscape, the coexpressed DElncRNA-DEmRNA networks were visualized. Functional annotation of the DEmRNAs co-expressed with DElncRNAs was performed with Metascape. A value of $p<$ 0.05 was set as the cut-off for significance.

\section{Validation in the Cancer genome atlas (TCGA) database}

The mRNA and lncRNA expression profiles of $171 \mathrm{pa}-$ tients with luminal B breast cancer and 59 normal tissues were downloaded from TCGA database to validate the expression patterns of selected DEmRNAs and DElncRNAs. 

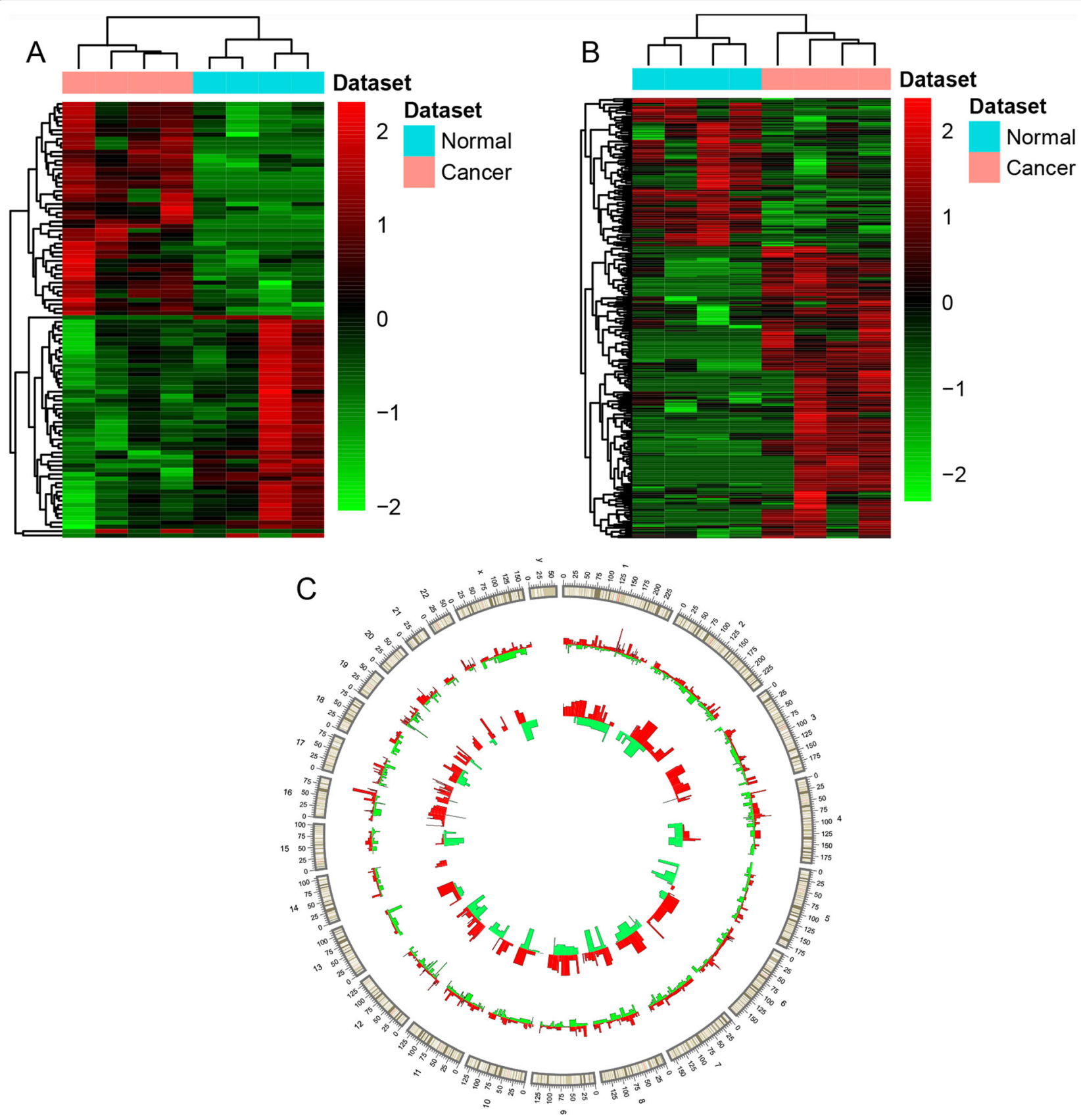

Fig. 1 DEIncRNAs and DEmRNAs between luminal-B breast cancer tumor tissues and adjacent tissues. $\mathbf{a}$ and $\mathbf{b}$ displayed hierarchical clustering results of top 100 DEmRNAs and DEIncRNAs between luminal-B breast cancer tumor tissues and adjacent tissues, respectively. Row and column represented DEmRNAs/DEIncRNAs and tissue samples, respectively. The color scale represented the expression levels. $\mathbf{c}$ displayed distribution of DElncRNAs and DEmRNAs on chromosomes. The outer layer cycle was the chromosome map of the human genome hg19 (GRCh37). The larger inner layer and smaller inner layer represented the distribution of DEmRNAs and DEIncRNAs on different chromosome, respectively. The red and green color represented the up- and down-regulated

\section{Results}

DEmRNAs and DElncRNAs between luminal-B breast cancer tumor tissues and adjacent tissues

A total of 1178 DEmRNAs (666 up-regulated and 512 down-regulated DEmRNAs) and 273 DElncRNAs (181 up-regulated and 92 down-regulated DElncRNAs) were obtained. The top 10 up- and down-regulated DEmRNAs and DElncRNAs were exhibited in Table 2 and Table 3, respectively. Hierarchical clustering analysis of top 10010 up- and down-regulated DEmRNAs and DElncRNAs was showed in Fig. 1a and Fig. 1b, respectively. Furthermore, the distribution of DElncRNAs and 


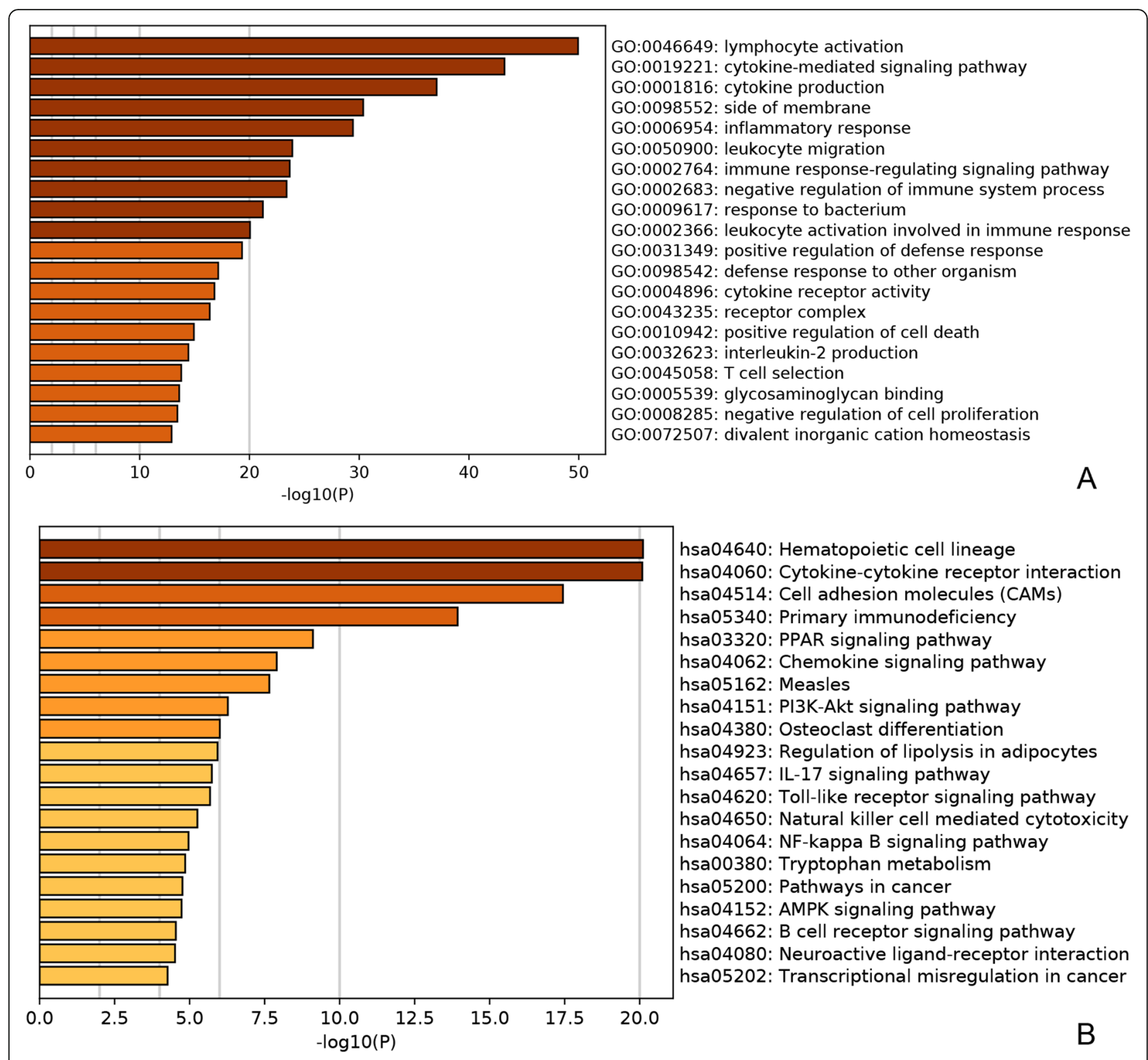

Fig. 2 Significantly enriched GO (a) terms and KEGG (b) pathways of DEmRNAs between luminal-B breast cancer tumor tissues and adjacent tissues. The $\mathrm{x}$-axis represented -lg $p$-value and the $\mathrm{y}$-axis shows GO terms or KEGG pathways

DEmRNAs on all chromosomes was showed in Fig. 1c. The raw-data have been uploaded to Gene Expression Omnibus (GEO) (GSE139274, https://www.ncbi.nlm.nih. gov/geo/query/acc.cgi?acc=GSE139274).

\section{Functional annotation}

Lymphocyte activation $(p=1.14 \mathrm{E}-50)$, cytokine-mediated signaling pathway $(p=5.74 \mathrm{E}-44)$ and cytokine production $(p=8.78 \mathrm{E}-38)$ were significantly enriched GO terms in luminal-B breast cancer (Fig. 2a). Hematopoietic cell lineage $(p=7.75 \mathrm{E}-21)$, Cytokine-cytokine receptor interaction $(p=8.19 \mathrm{E}-21)$, Cell adhesion molecules (CAMs) $(p=3.61 \mathrm{E}-18)$ and Primary immunodeficiency $(p=$
1.17E-14) were significantly enriched KEGG pathways in luminal-B breast cancer (Fig. 2b).

\section{Protein-protein interaction (PPI) networks}

The PPI networks included 281 nodes and 263 edges. FN1 $($ degree $=28)$, EGFR $($ degree $=14)$, JAK3 $($ degree $=$ $11)$, TUBB3 $($ degree $=11)$ and PTPRC $($ degree $=10)$ were five hub proteins of the PPI networks (Fig. 3).

\section{Cis-nearby-targeted DEmRNAs of DElncRNAs}

A total of 99 DElncRNAs-nearby-targeted DEmRNA pairs, involving in 78 DElncRNAs and 86 DEmRNAs, were detected (Fig. 4). Top two DElncRNAs with most 


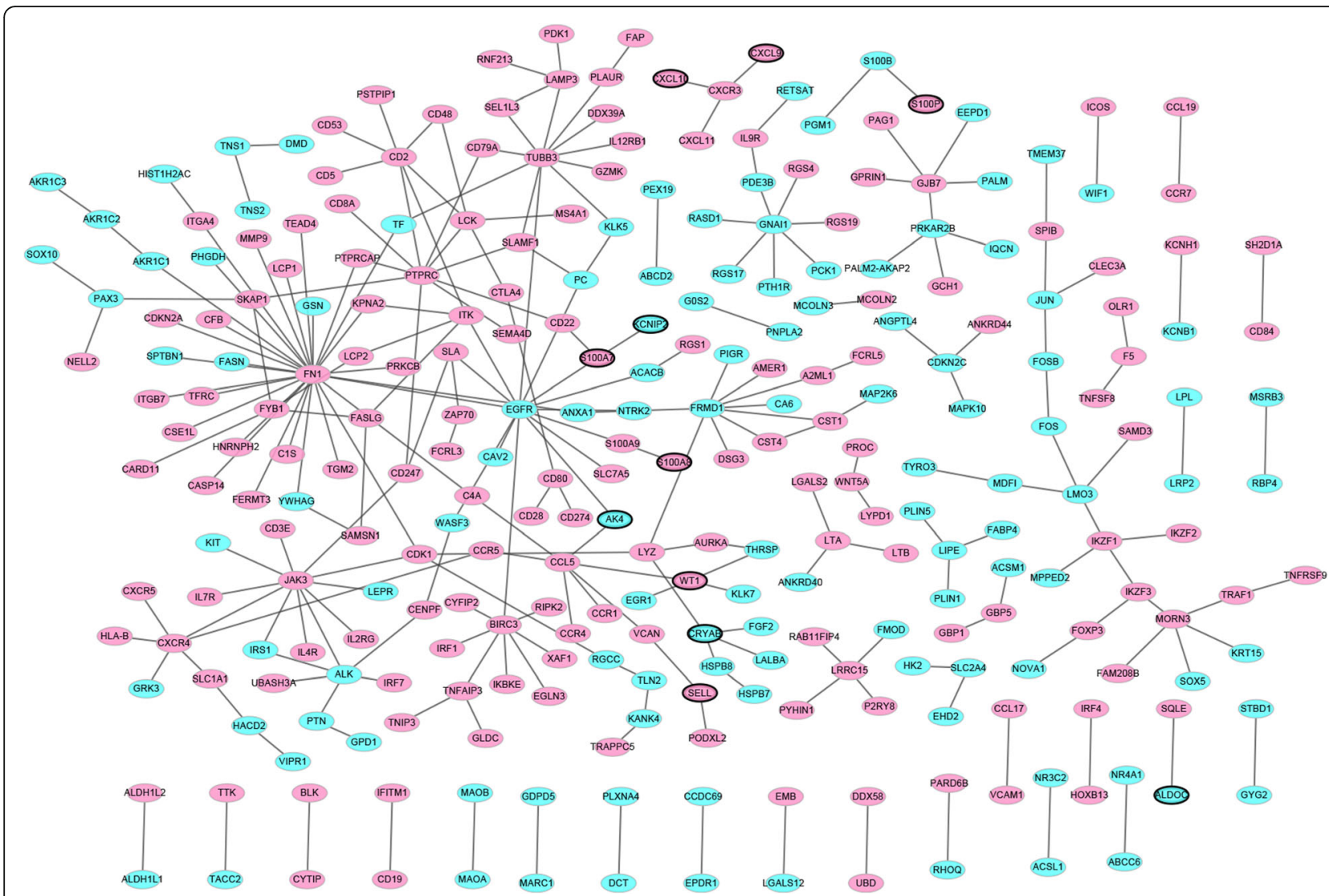

Fig. 3 Protein-protein interaction (PPI) networks. The red and blue ellipses represented proteins encoded by up- and down-regulated DEmRNAs between luminal-B breast cancer tumor tissues and adjacent tissues. Ellipses with black border were DEmRNAs derived from top 10 down- and up-regulated DEmRNAs between luminal-B breast cancer tumor tissues and adjacent tissues

nearby DEmRNAs were AL121985.1 and AL031316.1, which owned 5 and 4 nearby DEmRNAs, respectively. Regulation of cell adhesion $(p=1.84 \mathrm{E}-13)$, regulation of cell-cell adhesion $(p=7.86 \mathrm{E}-12)$, cytokine binding ( $p=$ 3.41E-07), $\mathrm{T}$ cell selection $(p=7.75 \mathrm{E}-07)$ and negative regulation of secretion $(p=1.38 \mathrm{E}-06)$ were significantly enriched GO terms (Fig. 6a). Cell adhesion molecules (CAMs) $\quad(p=1.72 \mathrm{E}-04), \quad$ Cytokine-cytokine receptor interaction $(p=2.81 \mathrm{E}-03)$, Primary immunodeficiency $(p=7.71 \mathrm{E}-03)$, AMPK signaling pathway $(p=9.30 \mathrm{E}-03)$ and Wnt signaling pathway $(p=1.46 \mathrm{E}-02)$ were significantly enriched KEGG pathways (Fig. 6b).

\section{DEIncRNA-DEmRNA co-expression networks}

A total of 1878 DElncRNA-DEmRNA co-expression pairs including 225 DElncRNAs and 737 DEmRNAs were obtained with an absolute value of PCC $>0.95$ and $p<0.01$ (Fig. 5).

Lymphocyte activation $(p=7.08 \mathrm{E}-50)$, cytokinemediated signaling pathway ( $p=9.35 \mathrm{E}-28)$, cytokine production $(p=1.68 \mathrm{E}-27)$, leukocyte migration $(p=3.20 \mathrm{E}-22)$ and alpha-beta $\mathrm{T}$ cell activation $(p=4.89 \mathrm{E}-20)$ were significantly enriched GO terms (Fig. 6c). Cytokine-cytokine receptor interaction $(p=2.18 \mathrm{E}-18)$, Hematopoietic cell lineage $(p=9.66 \mathrm{E}-16)$, Primary immunodeficiency $(p=$ $2.85 \mathrm{E}-15)$, Th1 and Th2 cell differentiation $(p=3.38 \mathrm{E}-13)$ and Cell adhesion molecules (CAMs) $(p=1.66 \mathrm{E}-12)$ were significantly enriched KEGG pathways (Fig. 6d).

\section{Validation in TCGA database}

With mRNA and lncRNA expression profiles downloaded from TCGA database, the expression patterns of four DEmRNAs, including S100A7, CCL5, MIAT and WT1-AS, were verified. As shown in Fig. 7, compared to normal controls, MIAT was down-regulated in luminal $\mathrm{B}$ breast cancer tumor tissues which were inconsistent with our results, while S100A7, CCL5 and WT1-AS were up-regulated in luminal B breast cancer tumor tissues which were consistent with our results.

\section{Discussion}

Breast cancer, as the most common non-cutaneous type of cancer, is the leading cause of cancer-related mortality among female globally (19). As luminal-B cancers showed poorer prognosis as compared to luminal-A cancers, we performed this study and identified abundant 


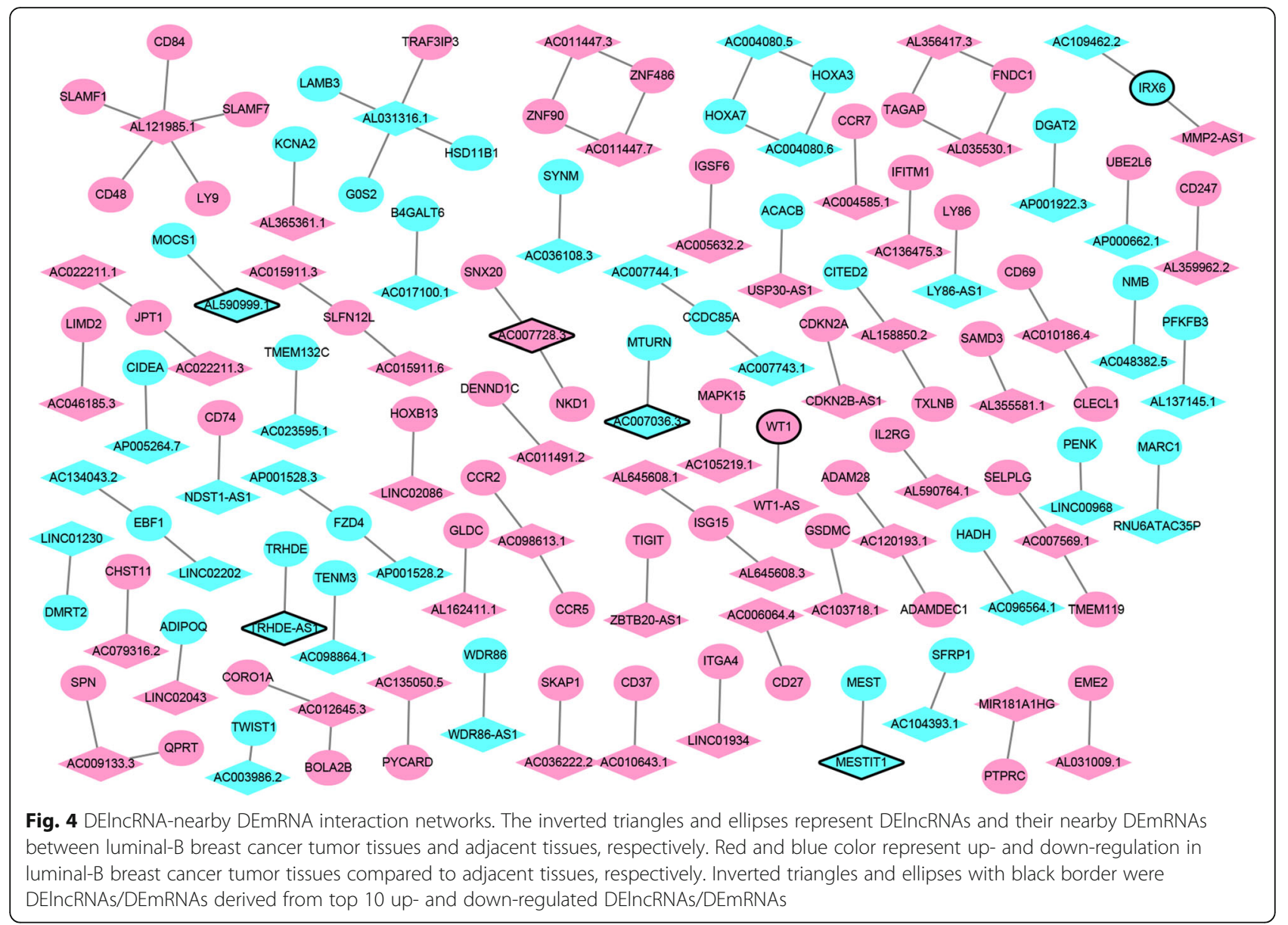

DElncRNAs and DEmRNAs between luminal-B breast cancer tumor tissues and adjacent tissues.

S100A7 is a member of the S100 protein family, which have been associated with preinvasive ductal carcinoma in situ (DCIS) (20). During breast tumorigenesis and/or progression, several S100 s, including S100A2, S100A4 and S100A7, exhibit altered expression levels based on molecular analysis of breast tumors (21). Cancemi et al. suggested that S100A7 was involved in critical phases of the breast cancer growth and progression (22). Mayama et al. proposed that S100A7 was linked to an aggressive phenotype of ER-positive breast carcinoma, and was potent marker for distant metastasis of ER-positive breast cancer patients (23). In current study, S100A7 was the most significant up-regulated DEmRNAs in luminal B breast cancer tumor tissues, which may indicated that S100A7 exert momentous roles in luminal B breast cancer.

Metastasis-associated lung adenocarcinoma transcript 1 (MALAT1) is a highly conserved lncRNA, and its overexpression in multiple cancerous tissues has been linked to the proliferation and metastasis of tumor cells. It was first identified as being up-regulated in lung tumors, and a prognostic marker for metastasis and patient survival in non-small cell lung cancer (NSCLC), specifically in early stages of lung adenocarcinoma (24). Subsequently, MALAT1 was shown to be up-regulated in a broad spectrum of tumor types, such as endometrial stromal sarcoma and hepatocellular carcinomas $(25,26)$. Additional, it has been found that MALAT1 gene mutations frequently occurred in luminal-type breast tumors (27). Besides, MALAT1 was one of the top 10 up-regulated DElncRNAs in this study, and co-expressed with S100A7, which emphasized the critical role the MALAT1 in luminal B breast cancer and suggested that MALAT1 may involve in luminal B breast cancer by regulating S100A7.

Chemokines, small-molecular-weight cytokines involved in the physiological control of immune cell migrationare, were reported to perform a crucial function in breast cancer tumorigenesis and progression (19). Recent years, the chemokine $\mathrm{C}-\mathrm{C}$ motif ligand 5 (CCL5), also known as RANTES, is a member of the CC subfamily, has been associated with aggressive breast cancer (28). Svensson et al. identified CCL2 and CCL5 as two therapeutic targets for estrogen-dependent breast 


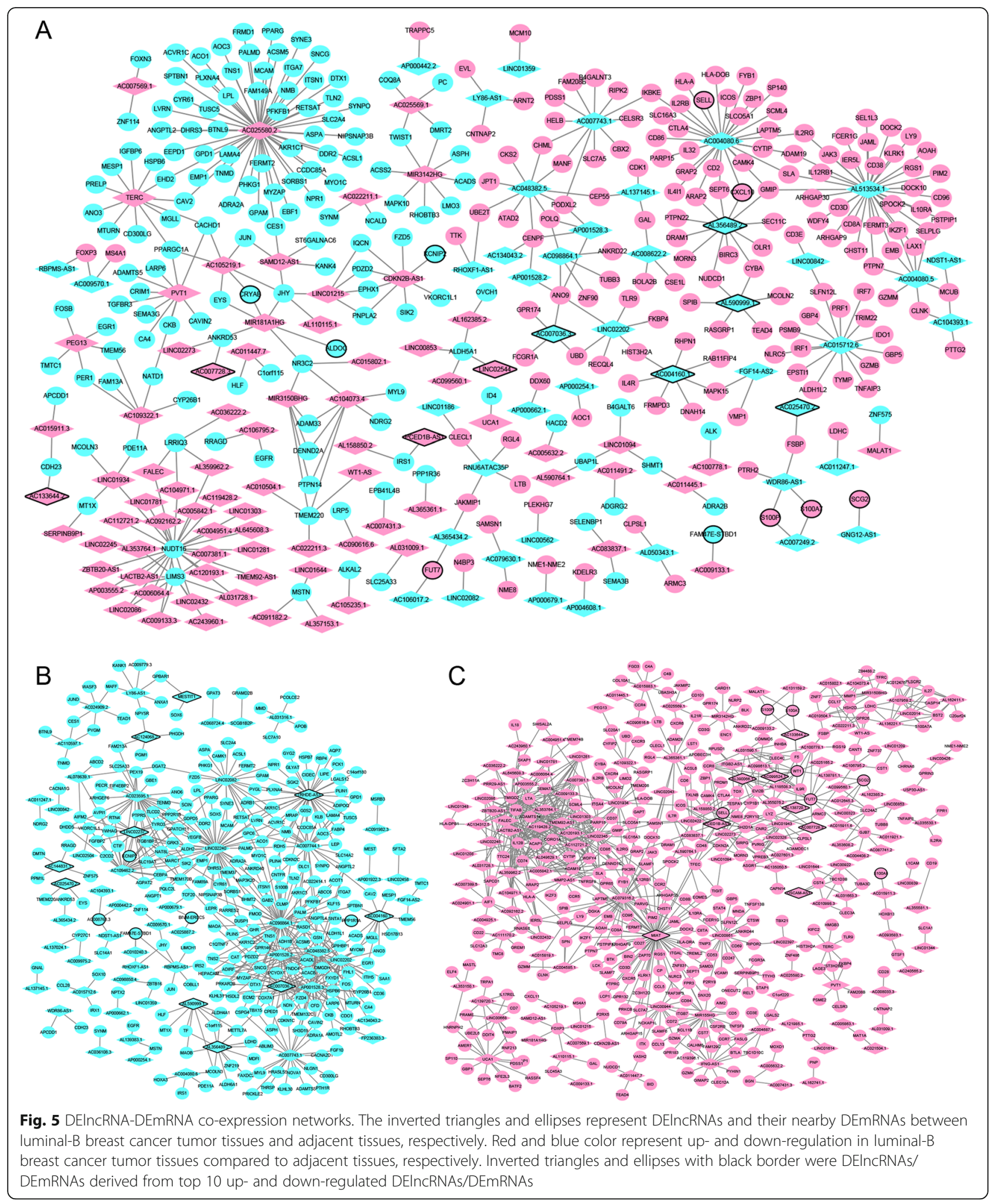

cancer (29). Previous study suggested that endothelial cells (ECs) enhance endothelial-mesenchymal transition (EMT)-induced triple-negative breast cancer (TNBC) cell metastasis through PAI-1 and CCL5 signaling (30).
Zhang et al. found that CCL5-mediated Th2 polarization of $\mathrm{CD}^{+}{ }^{+} \mathrm{T}$ cells promotes metastasis in luminal breast cancer (31). In our analysis, CCL5 was significant upregulated in luminal B breast cancer tumor tissues, 


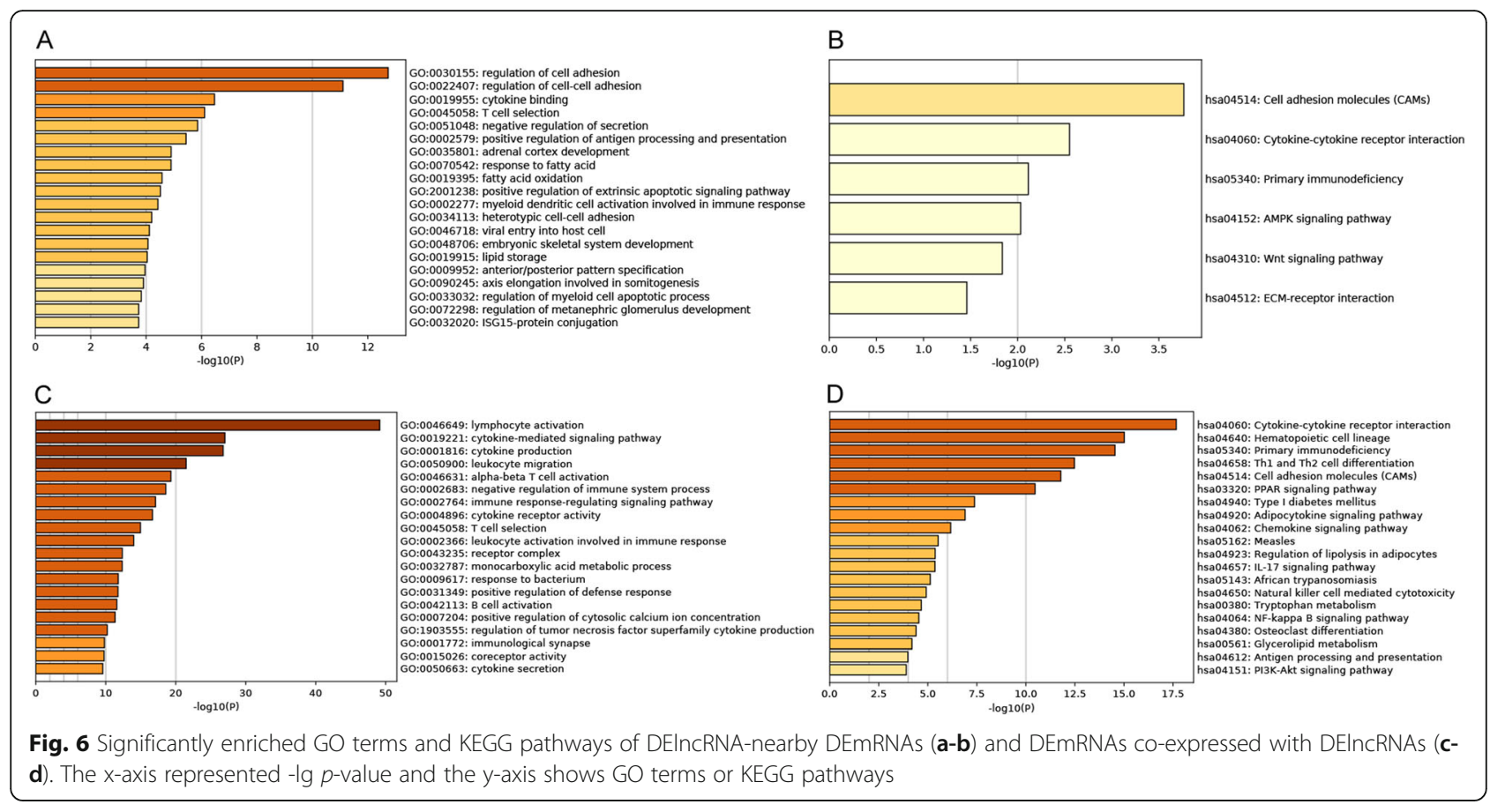

which indicated the key role of CCL5 in luminal B breast cancer.

LncRNA myocardial infarction-associated transcript (MIAT) is primarily expressed in heart and fetal brain tissue (32). Dysregulated MIAT was first reported to correlated with myocardial infarction and involved in cardiac hypertrophy and diabetic cardiomyopathy (3235). Recent studies suggested that MIAT promoted gastric cancer growth and metastasis by regulation of miR-141/DDX5 pathway, promoted proliferation and
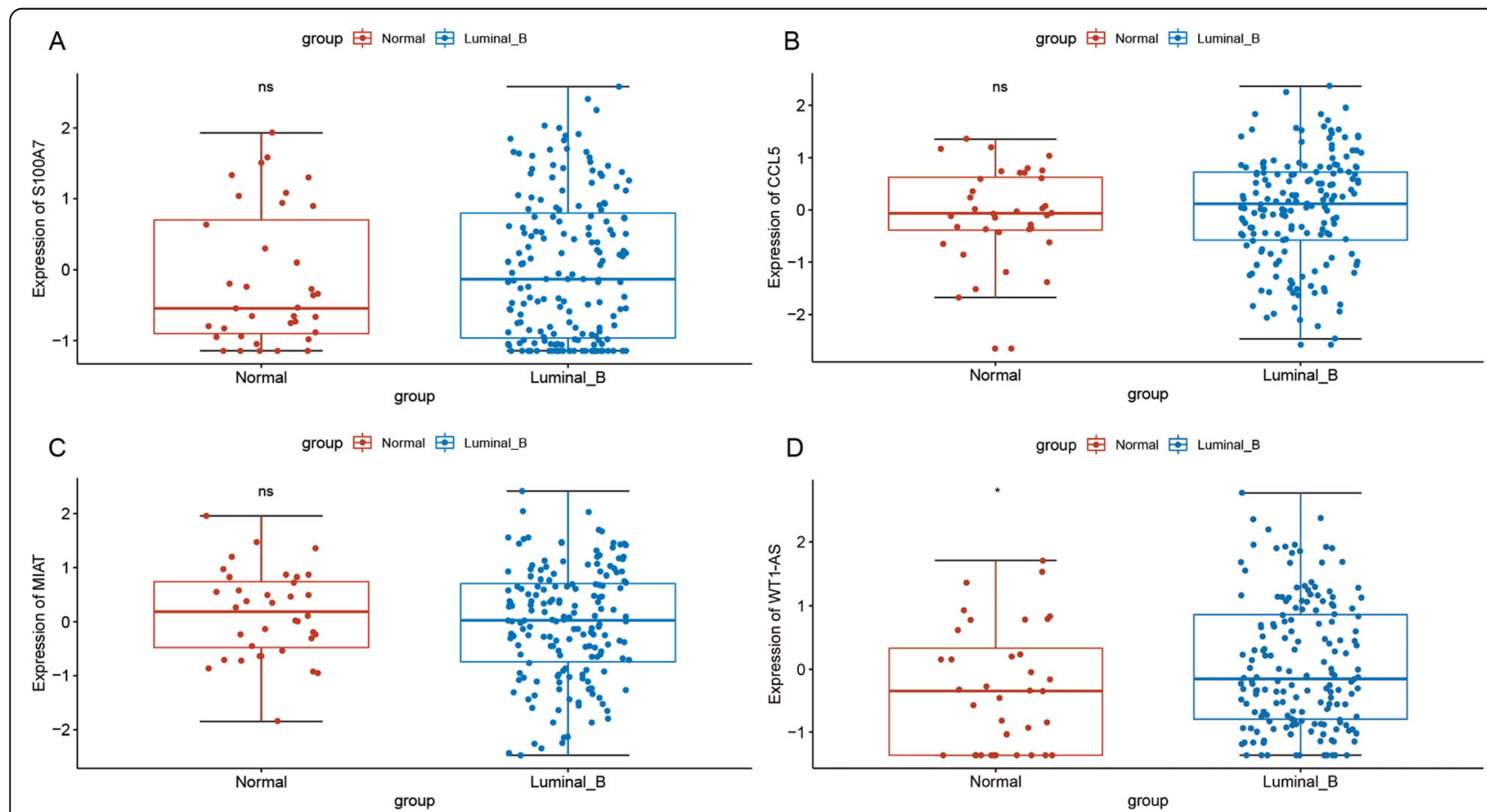

group 审 Normal 审 Luminal_B

Fig. 7 Validation in TCGA database. The x-axis shows luminal-B breast cancer tumor tissues and adjacent tissues, and the $y$-axis shows expression levels, respectively. A) S100A7; B) CCL5; C) MIAT; D) WT1-AS 
metastasis of non-small cell lung cancer via MMP9 activation, promoted hepatocellular carcinoma cells proliferation and invasion through sponging miR-214 (36-38). Luan et al. proposed that overexpression of MIAT was related to the TNM stage and lymphnode metastasis of breast cancer (39). MIAT was found to be overexpressed in both ER-positive breast cancer tissues and ER-positive breast cancer cell line MCF-7, and play a pivotal role in ER-positive breast cancer cell growth (40). Almnaseer-M et al. suggested that MIAT performs a critical function in breast tumorigenesis (41). MIAT was detected to be one of the top 10 up-regulated DElncRNAs, and coexpressed with CCL5. All these findings suggest that MIAT may involve in luminal B breast cancer by regulating the expression level of CCL5.

The Wilms' tumor 1 (WT1) was first cloned in 1990 as a suppressor in Wilms' tumor, which was located at chromosome 11p13 (42). WT1 gene mutations are linked with a subset of Wilm's tumors, the most common pediatric renal cancer (43). Substantial evidence has linked WT1 with the pathogenesis of breast cancer. WT1 is linked to the progression of breast cancer, including migration, invasion and angiogenesis. Knockdown of WT1 was demonstrated to lead to mitochondrial damage and then inhibit malignant cell growth (44). Highly expressed WT1 was linked to poor prognosis of patients with breast cancer (45). Over expression of WT1 was detected in TNBC (46). In agreement with previous studies, the expression of WT1 was observed significant up-regulated in luminal B breast cancer tumor tissues in present study.

Wilms tumor 1 Antisense RNA (WT1-AS) is located upstream of the WT1 gene, and these two genes are bidirectionally transcribed from the same promoter region. Down-regulation of WT1-AS was related to a poorer prognosis in ovarian clear cell adenocarcinoma (47). Lv et al. suggested that WT1-AS promoted cell apoptosis in hepatocellular carcinoma (HCC) and may function as a tumor suppressor in HCC (48). It is reported that WT1AS was significantly down-regulated in gastric cancers and may correlates with tumor progression (49). In addition, WT1-AS was detected to be was underexpressed in cervical carcinoma and suppress cervical cancer cell growth and aggressiveness $(50,51)$. In the current study, we found that WT1-AS was a DElncRNA and WT1 was a nearby-targeted DEmRNA of WT1-AS, which reminded us to explore the role of WT1-AS-WT1 in luminal B breast cancer.

\section{Conclusion}

In conclusion, a total of 1178 DEmRNAs and 273 DElncRNAs between luminal-B breast cancer tumor tissues and adjacent tissues were obtained. We discussed and emphasized the importance role of three
DElncRNA-DEmRNA pairs, including MALAT1S100A7, MIAT-CCL5 and WT1-AS-WT1, involved in luminal B breast cancer, which expected to provide new insight into understanding the mechanism underlying pathogenesis of luminal B breast cancer. The small sample size was a limitation of our study. Although the validation results in TCGA database indicated that our RNA-sequencing results were generally reliable, larger cohorts of patients and further experimental validation studies are needed to conduct to verify this conclusion.

\section{Abbreviations \\ CAMs: Cell adhesion molecules; CCL5: Chemokine C-C motif ligand 5; CNAs: Copy number alterations; DCIS: Ductal carcinoma in situ; DEIncRNA: Differentially expressed IncRNA; DEmRNA: Differentially expressed mRNA; ECs: Endothelial cells; EMT: Endothelial-mesenchymal transition; FPKM: Per million fragments mapped; GO: Gene Ontology; \\ HCC: Hepatocellular carcinoma; KEGG: Kyoto Encyclopedia of Genes and Genomes; IncRNAs: Long non-coding RNAs; MALAT1: Metastasis-associated lung adenocarcinoma transcript 1; MIAT: Myocardial infarction-associated transcript; ncRNAs: Non-coding RNAs; NSCLC: Non-small cell lung cancer; PCC: Pearson's correlation coefficient; PPI: Protein-protein interaction network; RIN: RNA integrity number; TNBC: Triple-negative breast cancer; WT1: Wilms' tumor 1; WT1-AS: Wilms tumor 1 Antisense RNA}

\section{Acknowledgements}

We are appreciated to Pro. Xin-Jian Jia for supplying the experimental samples.

\section{Authors' contributions}

CY and NZ2 made substantial contributions to conception and design. CY, $\mathrm{XJ}$ and $\mathrm{YY}$ performed the experiment. JE, NZ1 and XL collected and analyzed the data. WW and YL interpreted the data. All authors were involved in drafting and revising the manuscript and gave final approval of the manuscript.

\section{Funding}

This study was supported by Scientific Research Project of Sichuan Provincial Department of Health (090163). The funding body had no role in the design of the study and collection, analysis, and interpretation of data and in writing the manuscript.

\section{Availability of data and materials}

The raw-data have been uploaded to Gene Expression Omnibus (GEO) (GSE139274, https://www.ncbi.nlm.nih.gov/geo/query/acc.cgi?acc=GSE139274 ) and is publicly available until October 2020 due to data confidentiality.

Ethics approval and consent to participate

Written informed consent about the use of these samples was obtained from each patient. This study was approved by the ethical committee of People's Hospital of Deyang City (2017-045).

\section{Consent for publication}

Not applicable.

\section{Competing interests}

The authors declared that they have no competing interests.

\section{Author details}

'Department of Clinical Laboratory, People's Hospital of Deyang City, No. 173, Taishan North Road, Jingyang District, Deyang 618000, Sichuan, China. ${ }^{2}$ Department of Breast Surgery, People's Hospital of Deyang City, Deyang, China. ${ }^{3}$ Department of Science and Education, People's Hospital of Deyang City, Deyang, China. 
Received: 31 July 2019 Accepted: 22 November 2019 Published online: 03 December 2019

\section{References}

1. Torre LA, Bray F, Siegel RL, Ferlay J, Lortet-Tieulent J, Jemal A. Global cancer statistics, 2012. CA Cancer J Clin. 2015;65(2):87-108.

2. Parker JS, Michael M, Cheang MCU, Samuel L, David V, Tammi V, et al. Supervised risk predictor of breast cancer based on intrinsic subtypes. J Clin Oncol. 2009;27(8):1160.

3. Kornelia P. Heterogeneity in breast cancer. J Clin Investig. 2011;121(10): 3786-8

4. Untch M, Gerber B, Harbeck N, Jackisch C, Marschner N, Mobus V, et al. 13th st. Gallen international breast cancer conference 2013: primary therapy of early breast cancer evidence, controversies, consensus - opinion of a german team of experts (zurich 2013). Breast care (Basel, Switzerland). 2013; 8(3):221-9.

5. Weinstein JN, Collisson EA, Mills GB, Shaw KRM, Ozenberger BA, Kyle E, et al The Cancer genome atlas Pan-Cancer analysis project. Chin J Lung Cancer. 2015:45(10):1113-20

6. Cheng F, Oh DS, Lodewyk W, Britta W, Nuyten DSA, Nobel AB, et al. Concordance among gene-expression-based predictors for breast cancer. $\mathrm{N}$ Engl J Med. 2006;355(6):560.

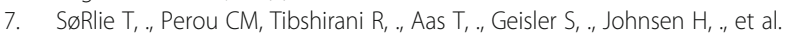
Gene expression patterns of breast carcinomas distinguish tumor subclasses with clinical implications. Proc Natl Acad Sci 2001.

8. Christos S, Lajos P. Gene-expression signatures in breast cancer. N Engl J Med. 2009;360(8):790.

9. Ponting $\mathrm{CP}$, Oliver $\mathrm{PL}$, Reik W. Evolution and functions of long noncoding RNAs. Cell. 2009;136(4):629-41.

10. Wilusz JE, Hongjae S, Spector DL. Long noncoding RNAs: functional surprises from the RNA world. Genes Dev. 2009;23(13):1494-504.

11. Wapinski $\mathrm{O}$, Chang HY. Long noncoding RNAs and human disease. Trends Cell Biol. 2011;21(6):354-61.

12. Francesco C, Akira W, Luca $Q$, Hui $X$, Larissa P, Abhijit $P$, et al. Identification of a long non-coding RNA as a novel biomarker and potential therapeutic target for metastatic prostate cancer. Oncotarget. 2014;5(3):764-74.

13. Zhuang M, Gao W, Xu J, Wang P, Shu Y. The long non-coding RNA H19derived miR-675 modulates human gastric cancer cell proliferation by targeting tumor suppressor RUNX1. Biochem Biophys Res Commun. 2014; 448(3):315-22

14. Wang Y, Wu N, Liu J, Wu Z, Dong D. FusionCancer: a database of cancer fusion genes derived from RNA-seq data. Diagn Pathol. 2015;10:131.

15. Mercer TR, Dinger ME, Mattick JS. Long non-coding RNAs: insights into functions. Nat Rev Genet. 2009:10(3):155-9.

16. Sun L, Li Y, Yang B. Downregulated long non-coding RNA MEG3 in breast cancer regulates proliferation, migration and invasion by depending on p53's transcriptional activity. Biochem Biophys Res Commun. 2016;478(1): 323-9.

17. Khaitan D, Dinger ME, Mazar J, Crawford J, Smith MA, Mattick JS, et al. The melanoma-upregulated long noncoding RNA SPRY4-IT1 modulates apoptosis and invasion. Cancer Res. 2011;71(11):3852-62.

18. Wang Y, Chen W, Yang C, Wu W, Wu S, Qin X, et al. Long non-coding RNA UCA1a(CUDR) promotes proliferation and tumorigenesis of bladder cancer. Int J Oncol. 2012;41(1):276-84.

19. Marco W, Wendy X, Pestell RG. The potential to target CCL5/CCR5 in breast cancer. Expert Opin Ther Targets. 2014;18(11):1265-75.

20. Liu H, Wang L, Wang X, Cao Z, Yang Q, Zhang K. S100A7 enhances invasion of human breast cancer MDA-MB-468 cells through activation of nuclear factor-kappaB signaling. World J Surg Oncol. 2013;11:93.

21. Emberley ED, Murphy LC, Watson PH. S100A7 and the progression of breast cancer. Breast Cancer Res. 2004;6(4):153-9.

22. Cancemi P, Di Cara G, Albanese NN, Costantini F, Marabeti MR, Musso R, et al. Differential occurrence of S100A7 in breast cancer tissues: a proteomic-based investigation. Proteomics Clin Appl. 2012;6(7-8):364-73.

23. Mayama A, Takagi K, Suzuki H, Sato A, Onodera Y, Miki Y, et al. OLFM4, LY6D and S100A7 as potent markers for distant metastasis in estrogen receptorpositive breast carcinoma. Cancer Sci. 2018;109(10):3350-9.

24. Ji P, Diederichs S, Wang W, Boing S, Metzger R, Schneider PM, et al. MALAT1 , a novel noncoding RNA, and thymosin beta4 predict metastasis and survival in early-stage non-small cell lung cancer. Oncogene. 2003;22(39): $8031-41$.
25. Yamada K, Kano J, Tsunoda H, Yoshikawa H, Okubo C, Ishiyama T, et al. Phenotypic characterization of endometrial stromal sarcoma of the uterus. Cancer Sci. 2006;97(2):106-12.

26. Lin R, Maeda S, Liu C, Karin M, Edgington TS. A large noncoding RNA is a marker for murine hepatocellular carcinomas and a spectrum of human carcinomas. Oncogene. 2007;26(6):851-8.

27. Ellis MJ, Ding L, Shen D, Luo J, Suman VJ, Wallis JW, et al. Whole-genome analysis informs breast cancer response to aromatase inhibition. Nature. 2012:486(7403):353-60.

28. Ayesha K, Joy W, llaria F, Chaofeng M, Junhua M, Zhizhou Y, et al. Recent Advances in Discovering the Role of CCL5 in Metastatic Breast Cancer. Mini Reviews in Medicinal Chemistry. 2016;15(13).

29. Svensson S, Abrahamsson A, Rodriguez GV, Olsson AK, Jensen L, Cao Y, et al. CCL2 and CCL5 are novel therapeutic targets for estrogen-dependent breast Cancer. Clin Cancer Res. 2015:21(16):3794-805.

30. Zhang W, Xu J, Fang H, Tang L, Chen W, Sun Q, et al. Endothelial cells promote triple-negative breast cancer cell metastasis via PAI-1 and CCL5 signaling. Faseb Journal Official Publication of the Federation of American Societies for Experimental Biology. 2017;32(1):fj.201700237RR.

31. Qianfei Z, Jilong Q, Lin Z, Lei G, Bing Z, Yan Z, et al. CCL5-mediated Th2 immune polarization promotes metastasis in luminal breast Cancer. Cancer Res. 2015;75(20):4312-21.

32. Zhu XH, Yuan YX, Rao SL, Wang P. LncRNA MIAT enhances cardiac hypertrophy partly through sponging miR-150. Euro Rev Med Pharmacol Sci. 2016;20(17):3653.

33. Zhou X, Zhang W, Jin M, Chen J, Xu W, Kong X. IncRNA MIAT functions as a competing endogenous RNA to upregulate DAPK2 by sponging miR-22-3p in diabetic cardiomyopathy. Cell Death Dis. 2017;8(7):e2929.

34. Li Y, Wang J, Sun L, Zhu S. LncRNA myocardial infarction-associated transcript (MIAT) contributed to cardiac hypertrophy by regulating TLR4 via miR-93. Eur J Pharmacol. 2017:818:508-17.

35. Ishii N, Ozaki K, Sato H, Mizuno H, Saito S, Takahashi A, et al. Identification of a novel non-coding RNA, MIAT, that confers risk of myocardial infarction. J Hum Genet. 2006:51(12):1087-99.

36. Sha M, Lin M, Wang J, Ye J, Xu J, Xu N, et al. Long non-coding RNA MIAT promotes gastric cancer growth and metastasis through regulation of miR-141/DDX5 pathway. Journal of experimental \& clinical cancer research : CR. 2018;37(1):58.

37. Lai I, Yang CA, Lin PC, Chan WL, Lee YT, Yen JC, et al. Long noncoding RNA MIAT promotes non-small cell lung cancer proliferation and metastasis through MMP9 activation. Oncotarget. 2017:8(58):98148-62.

38. Huang X, Gao Y, Qin J, Lu S. IncRNA MIAT promotes proliferation and invasion of HCC cells via sponging miR-214. Am J Physiol Gastrointest Liver Physiol. 2018;314(5):G559-g65.

39. Luan T, Zhang X, Wang S, Song Y, Zhou S, Lin J, et al. Long non-coding RNA MIAT promotes breast cancer progression and functions as ceRNA to regulate DUSP7 expression by sponging miR-155-5p. Oncotarget. 2017;8(44):76153-64.

40. Li Y, Jiang B, Wu X, Huang $Q$, Chen W, Zhu H, et al. Long non-coding RNA MIAT is estrogen-responsive and promotes estrogen-induced proliferation in ER-positive breast cancer cells. Biochem Biophys Res Commun. 2018; 503(1):45-50.

41. Almnaseer ZA, Mourtada-Maarabouni M. Long noncoding RNA MIAT regulates apoptosis and the apoptotic response to chemotherapeutic agents in breast cancer cell lines. Biosci Rep. 2018;38(4).

42. Call KM, Glaser T, Ito CY, Buckler AJ, Pelletier J, Haber DA, et al. Isolation and characterization of a zinc finger polypeptide gene at the human chromosome 11 Wilms' tumor locus. Cell. 1990;60(3):509-20.

43. Silberstein GB, Van Horn K, Strickland P, Roberts CT Jr, Daniel CW. Altered expression of the WT1 wilms tumor suppressor gene in human breast cancer. Proc Natl Acad Sci U S A. 1997;94(15):8132-7.

44. Tatsumi N, Oji Y, Tsuji N, Tsuda A, Higashio M, Aoyagi S, et al. Wilms' tumor gene WT1-shRNA as a potent apoptosis-inducing agent for solid tumors. Int J Oncol. 2008:32(3):701-11.

45. Yasuo M, Akiko A, Chiyomi E, Tetsuya T, Yasuhiro T, Hiroya T, et al. High expression of Wilms' tumor suppressor gene predicts poor prognosis in breast cancer patients. Clin Cancer Res. 2002;8(5):1167.

46. Xiao-Wei Q, Fan Z, Xin-Hua Y, Lin-Jun F, Yi Z, Yan L, et al. High Wilms' tumor 1 mRNA expression correlates with basal-like and ERBB2 molecular subtypes and poor prognosis of breast cancer. Oncol Rep. 2012;28(4):1231-6.

47. Kaneuchi M, Sasaki M, Tanaka Y, Shiina H, Yamada H, Yamamoto R, et al. WT1 and WT1-AS genes are inactivated by promoter methylation in ovarian clear cell adenocarcinoma. Cancer. 2005;104(9):1924-30. 
48. Lv L, Chen G, Zhou J, Li J, Gong J. WT1-AS promotes cell apoptosis in hepatocellular carcinoma through down-regulating of WT1. J Exp Clin Cancer Res : CR. 2015;34:119.

49. Du T, Zhang B, Zhang S, Jiang X, Zheng P, Li J, et al. Decreased expression of long non-coding RNA WT1-AS promotes cell proliferation and invasion in gastric cancer. Biochim Biophys Acta. 2016;1862(1):12-9.

50. Cui L, Nai M, Zhang K, Li L, Li R. IncRNA WT1-AS inhibits the aggressiveness of cervical cancer cell via regulating p53 expression via sponging miR-3305p. Cancer Manag Res. 2019;11:651-67.

51. Dai SG, Guo LL, Xia X, Pan Y. Long non-coding RNA WT1-AS inhibits cell aggressiveness via miR-203a-5p/FOXN2 axis and is associated with prognosis in cervical cancer. Eur Rev Med Pharmacol Sci. 2019;23(2):486-95.

\section{Publisher's Note}

Springer Nature remains neutral with regard to jurisdictional claims in published maps and institutional affiliations.

Ready to submit your research? Choose BMC and benefit from:

- fast, convenient online submission

- thorough peer review by experienced researchers in your field

- rapid publication on acceptance

- support for research data, including large and complex data types

- gold Open Access which fosters wider collaboration and increased citations

- maximum visibility for your research: over $100 \mathrm{M}$ website views per year

At $\mathrm{BMC}$, research is always in progress.

Learn more biomedcentral.com/submissions 